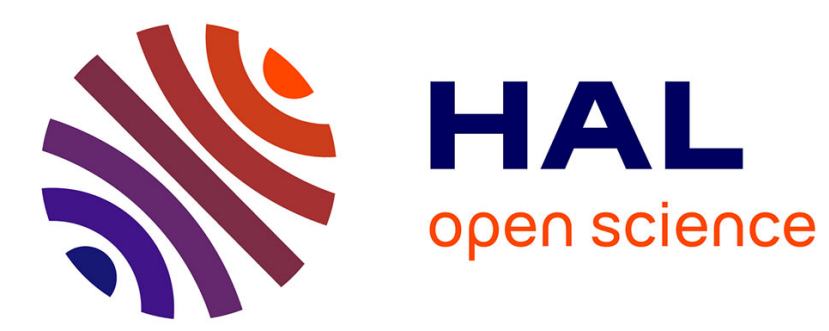

\title{
LA CUISINE, UN LIEU ET SES PRATIQUES. DE LA TRADITION À LA TRANSGRESSION
}

Crystel Pinçonnat

\section{To cite this version:}

Crystel Pinçonnat. LA CUISINE, UN LIEU ET SES PRATIQUES. DE LA TRADITION À LA TRANSGRESSION. Revue de Litterature Comparee, 2010, 3 (335), pp.305-318. hal-01382538

\section{HAL Id: hal-01382538 \\ https://hal.science/hal-01382538}

Submitted on 19 Oct 2016

HAL is a multi-disciplinary open access archive for the deposit and dissemination of scientific research documents, whether they are published or not. The documents may come from teaching and research institutions in France or abroad, or from public or private research centers.
L'archive ouverte pluridisciplinaire HAL, est destinée au dépôt et à la diffusion de documents scientifiques de niveau recherche, publiés ou non, émanant des établissements d'enseignement et de recherche français ou étrangers, des laboratoires publics ou privés. 


\title{
LA CUISINE, UN LIEU ET SES PRATIQUES. DE LA TRADITION À LA TRANSGRESSION
}

\author{
Crystel Pinçonnat
}

Klincksieck I Revue de littérature comparée

$2010 / 3-n^{\circ} 335$

pages 305 à 318

\section{ISSN 0035-1466}

Article disponible en ligne à l'adresse:

http://www.cairn.info/revue-de-litterature-comparee-2010-3-page-305.htm

Pour citer cet article :

Pinçonnat Crystel , « La cuisine, un lieu et ses pratiques. De la tradition à la transgression », Revue de littérature comparée, 2010/3 n³35, p. 305-318.

Distribution électronique Cairn.info pour Klincksieck.

(c) Klincksieck. Tous droits réservés pour tous pays.

La reproduction ou représentation de cet article, notamment par photocopie, n'est autorisée que dans les limites des conditions générales d'utilisation du site ou, le cas échéant, des conditions générales de la licence souscrite par votre établissement. Toute autre reproduction ou représentation, en tout ou partie, sous quelque forme et de quelque manière que ce soit, est interdite sauf accord préalable et écrit de l'éditeur, en dehors des cas prévus par la législation en vigueur en France. II est précisé que son stockage dans une base de données est également interdit. 


\section{La cuisine, un lieu et ses pratiques De la tradition à la transgression}

L'espace de la cuisine intéresse généralement peu la littérature. Typiquement, « la topographie disparaît derrière l'action $»^{1}$ : on s'attache plutôt à l'activité du personnage dans la cuisine, activité qui, dans sa nature de «théâtre de gestes » ${ }^{2}$, occulte son cadre tout en le sous-entendant. Autant les cuisines de ferme ou de tout autre univers exotique, sources de dépaysement pour le lecteur citadin, possèdent des charmes pittoresques qui invitent à la description, autant la cuisine de la domesticité ordinaire ne présente - dans sa quotidienneté du moins - guère d'attrait romanesque.

\section{Cuisine et tradition}

Avant d'aborder plus en détail le $X X^{e}$ siècle, quelques pratiques romanesques - révélatrices de procédés récurrents dans la tradition - méritent toutefois d'être relevées. Au XVII et au XVIII ${ }^{\mathrm{e}}$ siècles, la cuisine est un lieu ambigu au sein de la maison des maîtres, un espace à la fois ouvert et fermé qui reflète l'ordre social du fait de la distribution spatiale qu'elle opère. Elle intervient comme une sorte de seuil, de sas discriminatoire au sein du foyer. On y distingue le peuple des livreurs qui y entre sans pénétrer plus avant dans l'intimité de la maison, la domesticité qui s'y affaire pour le plus grand plaisir des maîtres, et ceux - hôtes et convives - qui sont parfois invités à la traverser, décryptant dans son spectacle la promesse de futures agapes:

[...] nous n'apperçumes pas sans plaisir dans une cuisine garnie de tous les ustanciles necessaires trois personnes occupées à prépa-

1. Je reprends ici, tout en l'inversant, la formule de Roman Jakobson : « l'action disparaît derrière la topographie » (« Notes marginales sur la prose de Pasternak », Huit questions de poétique, Paris, Seuil, 1977, p. 68).

2. Jean Baudrillard, Le Système des objets, Paris, Gallimard, « Tel », 1968, p. 79. 
rer notre souper, c'est-à-dire, un cuisinier, un aide-de-cuisine et un foüille-au-pot. ${ }^{3}$

Dans la demeure, la cuisine est un lieu intermédiaire, qui définit une certaine distribution. C'est, d'une part, un espace de travail où les aliments sont rendus propres à la consommation. Et c'est, d'autre part, un lieu de passage où statut social et mobilité dépendent étroitement de la place assignée à chacun au sein du processus de traitement de la nourriture. À l'extérieur, au plus près du cru, se tient le peuple. La salle à manger, l'au-delà intime de la cuisine, où s'effectue la consommation et l'ingestion, est l'espace réservé aux convives et aux maîtres. Entre les deux : se trouve la cuisine, soit le monde des «passeurs», cuisiniers, marmitons et serviteurs. Le rituel des repas définit donc une circulation des corps, organisée pour les corps au sein de la maison aristocratique ou bourgeoise, dont la cuisine est le cœur. De ce fait, si on la mentionne fréquemment, la cuisine intéresse généralement aussi peu que les personnages de second plan qui l'occupent, personnages que leur association - parfois erronée - avec cet univers, condamnent au statut de subalternes tant du point de vue de la hiérarchie sociale que de l'intérêt romanesque :

Que nous ferons bonne chère, nous mangerons à la table du roi, mais pour Finette elle lavera les écuelles dans la cuisine, car elle est faite comme une souillon, et si l'on demande qui elle est, gardons-nous bien de l'appeler notre sœur : il faudra dire que c'est la petite vachère du village. ${ }^{4}$

Au XIXe siècle cependant, en accord avec « l'intérêt pour tout ce qui est "bas" : vie populaire, vie domestique, vie charnelle [que] le réalisme ambitionne d'instaurer [...] en nouveaux territoires du roman $»^{5}$, des descriptions plus étoffées de cuisines commencent à apparaître. Si elles valorisent un lieu de l'activité humaine marginalisé jusqu'alors, elles n'en répondent pas moins au principe de mise en équivalence entre le personnage et son lieu, entre l'habitant et son habitat qui caractérise le réalisme. «Montre-moi où tu vis, je te dirai qui tu es ${ }^{6}{ }^{6}$, écrit Jakobson. Au début du Père Goriot, le personnage de Madame Vauquer illustre ce principe phare du réalisme :

[...] toute sa personne explique la pension, comme la pension implique sa personne. [...] Son jupon [...] dont la ouate s'échappe par les fentes de

3. Alain-René Lesage, Le Bachelier de Salamanque ou les mémoires et avantures de Don de Chérubin de la Ronda, Paris, Valleyre, 1736, p. 219.

4. Marie-Catherine d'Aulnoy, «Finette Cendron », Le Cabinet des fées ou Collection choisie des contes des fées et autres contes merveilleux, Paris, Barbin, 1698, p. 500.

5. Jacques Dubois, Les Romanciers du réel, Paris, Seuil, «Points/Essais », 2000, p. 49.

6. Roman Jakobson, art. cité, p. 68. 
l'étoffe lézardée, résume le salon, la salle à manger, le jardinet, annonce la cuisine et fait pressentir les pensionnaires. ${ }^{7}$

C'est dans cette même perspective que Balzac décrit assez longuement la cuisine des Topinard dans Le Cousin Pons :

Le logement de Topinard consistait en une cuisine et en deux chambres. [...] Les riches n'imagineraient pas la simplicité de la batterie de cuisine qui consistait en une cuisinière, un chaudron, un gril, une casserole, deux ou trois marabouts, et une poêle à frire. La vaisselle en faïence, brune et blanche, valait bien douze francs. La table servait à la fois de table de cuisine et de table à manger. Le mobilier consistait en deux chaises et deux tabourets. [...] Et dans un coin s'élevait le baquet où se savonnait, souvent pendant la nuit, le linge de la maison. [...] On conçoit, sur ce léger croquis, que les Topinard étaient, selon la phrase devenue proverbiale, pauvres mais honnêtes. ${ }^{8}$

Ici, la description épuise son objet sous la forme à peine camouflée d'un état des lieux et d'un inventaire. Tous les détails sont redondants et ne signifient qu'une seule et même chose : la pauvreté du ménage.

\section{Régimes satirique et ironique}

Le $X X^{e}$ siècle tend à prolonger cette tendance. Objet de consommation formaté dans l'imaginaire collectif par les magazines et la publicité, la cuisine est souvent décrite à des fins satiriques. Ces descriptions tiennent lieu d'indicateur social, elles stigmatisent une classe sociale, un certain mode de vie. Dans cette perspective, on songe aux Choses de Georges Perec, soustitré Une histoire des années soixante. Dans le premier chapitre, la cuisine de l'appartement rêvé par le jeune couple est ironiquement décrite au conditionnel à valeur d'irréel :

Il y aurait une cuisine vaste et claire, avec des carreaux bleus armoriés, trois assiettes de faïence décorées d'arabesques jaunes, à reflets métalliques, des placards partout, une belle table de bois blanc au centre, des tabourets, des bancs. Il serait agréable de venir s'y asseoir, chaque matin, après une douche, à peine habillé. Il y aurait sur la table un gros beurrier de grès, des pots de marmelade, du miel, des toasts, des pamplemousses coupés en deux. ${ }^{9}$

7. Honoré de Balzac, Le Père Goriot, 1843, La Comédie humaine, Paris, Gallimard, t. III, «Bibliothèque de la Pléiade », 1973, p. 54-55.

8. Honoré de Balzac, Le Cousin Pons, 1846, La Comédie humaine, Paris, Gallimard, t. VII, « Bibliothèque de la Pléiade », 1977, p. 751-752.

9. Georges Perec, Les Choses, une histoire des années soixante, Paris, Julliard, 1965, «Presses Pocket », p. 14-15. 
Difficile de ne pas lire dans cette description un pastiche des cuisines reproduites dans certains magazines féminins. Cette description évoque quelque peu le rêve de la maison d'enfance telle que la conçoit Bachelard, un lieu tout d'équilibre, de paix et de bonheur domestiques ${ }^{10}$ qui se décryptent dans la fidélité au passé : détails renvoyant à une certaine rusticité (« carreaux bleus armoriés », « assiettes de faïence », « beurrier de grès »), éléments auxquels s'ajoutent humoristiquement des signes de modernité et de distinction (« belle table de bois blanc», «toasts », «pamplemousses coupés en deux»). L'ironie de cette description éclate, lorsqu'au chapitre suivant, Perec décrit l'appartement où le jeune couple est contraint de vivre, faute de revenus conséquents:

Pour une superficie totale de trente-cinq mètres carrés, [...] leur appartement se composait d'une entrée minuscule, d'une cuisine exiguë, dont une moitié avait été aménagée en salle d'eau, d'une chambre aux dimensions modestes, d'une pièce à tout faire [...] et d'un coin mal défini, à mi-chemin du cagibi et du corridor, où parvenaient à prendre place un réfrigérateur de petit format, un chauffe-eau électrique, une penderie de fortune, une table, où ils prenaient leur repas, et un coffre à linge sale qui leur servait également de banc. ${ }^{11}$

C'en est fini du beau rêve dans lequel un espace spécifique était consacré à chacune des activités les plus ordinaires de la vie quotidienne. L'ordre familial et son assise, que signalaient la position centrale de la table ainsi que l'usage spécifique auquel chaque objet était destiné, se défont. Dans l'appartement fourre-tout, pièces et objets sont multi-fonctionnels: la cuisine fait également office de salle d'eau, ses constituants traditionnels s'éparpillent dans des espaces indéfinis, la table et le réfrigérateur sont relégués dans le cagibi-corridor. Après l'utopie des rêveries grandioses, on comprend bien vite que la «guerre d'usure », engendrée par la tyrannie de l'espace et le sentiment de frustration, l'emportera rapidement sur les plaisirs temporaires de la vie de bohème.

Dans les années cinquante, Boris Vian a lui aussi violemment dénoncé le conformisme matériel d'une certaine jeunesse. On connaît l'humour grinçant avec lequel il ridiculisait la vénalité amoureuse de la nouvelle génération, dans sa chanson intitulée Complainte du progrès :

Autrefois pour faire sa cour

On parlait d'amour [...]

Aujourd'hui, c'est plus pareil [...]

Pour séduire le cher ange

On lui glisse à l'oreille

Ah... Gudule !... Viens m'embrasser... Et je te donnerai [...]

Une cuisinière

10. Gaston Bachelard, La Poétique de l'espace [1957], Paris, PUF, 1974, p. 30.

11. Georges Perec, Les Choses, op. cit., p. 20. 


\begin{abstract}
Avec un four en verre
Des tas de couverts

Et des pell'à gâteaux ${ }^{12}$
\end{abstract}

Peu de trace de cette satire de la vie moderne subsiste dans L'Écume des jours. Dans l'appartement où vit Colin, la cuisine est un lieu convivial d'harmonie et de raffinement, dont la modernité, loin de déshumaniser ses usagers, répond au principe de plaisir :

Le couloir de la cuisine était clair, vitré des deux côtés, et un soleil brillait de chaque côté, car Colin aimait la lumière. Il y avait des robinets de laiton soigneusement astiqués, un peu partout. Les jeux de soleil sur les robinets produisaient des effets féeriques. [...] Colin poussa la porte émaillée de la cuisine. Le cuisinier Nicolas surveillait un tableau de bord. Il était assis devant son pupitre également émaillé de clair jaune et qui portaient des cadrans correspondant aux divers appareils culinaires alignés le long des murs. L'aiguille du four électrique réglé pour la dinde rôtie, oscillait entre «presque » et « à point ». ${ }^{13}$

Pourtant bien vite, cet univers harmonieux se disloque. L'appartement s'amenuise, se rétrécit, envahi lui aussi - au fur et à mesure que le nénuphar ronge les poumons de Chloé - par une morbidité maladive :

À travers les vitres, de chaque côté, on distinguait un soleil terne, blafard, semé de grandes taches noires, un peu plus lumineux en son centre. Quelques maigres faisceaux de rayons réussissaient à pénétrer dans le couloir, mais, au contact des carreaux de céramique autrefois si brillants, ils se fluidifiaient et ruisselaient en longues traces humides. Une odeur de cave émanait des murs. ${ }^{14}$

Sur un mode poétique et merveilleux, on retrouve donc, dans L'Écume des jours, l'homologie qui caractérisait le personnage et son lieu dans le roman réaliste. La cuisine, espace vital de régénération de l'être que symbolisaient la lumière, le soleil et la nourriture, extériorise et matérialise désormais, au sein d'une maison devenue elle aussi mortifère, l'étouffement des personnages, l'un asphyxié par la maladie et les autres, par le manque d'argent.

Maître des exercices de style, Italo Calvino exploite, lui aussi, ce rapport spéculaire entre personnage et cuisine. Dans Se una notte d'inverno un viaggiatore (Si par une nuit d'hiver un voyageur), la parodie consiste à reprendre une scène assez récurrente dans le roman policier : l'enquêteur décrypte la personnalité du suspect en inventoriant le contenu de sa cuisine et de son réfrigérateur. Ici toutefois, le jeu est plus complexe puisque c'est le Lecteur, soit le protagoniste du roman, qui se livre à cette activité pour tenter de

12. Boris Vian, Textes et chansons, Paris, René Julliard, «10/18 », 1966, p. 84-85.

13. Boris Vian, L'Écume des jours, Paris, J.-J. Pauvert, «10/18 », 1963, p. 10-11.

14. Ibid., p. 120. 
mieux saisir le mystérieux personnage féminin qui l'intrigue et le fascine : la Lectrice.

La cucina è la parte della casa che può dire più cose di te : se fai da mangiare o no (si direbbe di si, se non tutti i giorni, abbastanza regolarmente), se per te sola $o$ anche per altri [...] se tendi al minimo indispensabile o alla gastronomia [...] se stare ai fornelli rappresenta per te una penosa necessità o anche un piacere [...]. Un'occhiata al frigorifero può permettere di raccogliere altri dati preziosi : nei palchetti portauova c'è rimasto un solo uovo; di limoni ce n'è solo mezzo e mezzo secco; insomma, nei rifornimenti essenziali si nota qualche trascuratezza. In compenso c'è crema di marroni, olive nere, un vasetto di salsifis o scorzo bianca : è chiaro che nel far la spesa ti lasci attrarre dalle merci che vedi esposte, più che avere in mente ciò che manca in casa.

Osservando la tua cucina dunque si può ricavare una immagine di te come donna estroversa e lucida, sensuale e metodica, che mette il senso pratico al servizio della fantasia. ${ }^{15}$

Paradoxalement, le portrait de la Lectrice ne s'effectue pas - comme c'est fréquemment le cas dans la tradition - par la description du contenu de sa bibliothèque, mais par celui de sa cuisine. Pour autant, ce passage accomplit humoristiquement la même fonction: celle de révélateur du personnage.

\section{Cuisines et transgressions}

Par-delà ces descriptions anthropocentriques, «au service du personnage ${ }^{16}$, qui découlent d'une esthétique réaliste, mais se jouent parfois de ses conventions, j'aimerais m'intéresser aux descriptions de cuisines qui s'étoffent, me semble-t-il, dès lors que ce lieu devient le cadre d'une

15. Italo Calvino, Se una notte d'inverno un viaggiatore, Turin, Einaudi, 1979, p. 143-144; traduction française de Danièle Sallenave et François Wahl : « La cuisine est la partie de la maison qui peut dire le plus de choses sur toi : si tu te fais à manger ou non lon dirait que oui, pas tous les jours, mais fréquemment), si c'est pour toi toute seule ou pour d'autres aussi [...], si tu te limites au minimum, à l'indispensable, ou si tu as du goût pour la gastronomie [...] si c'est pour toi une nécessité pénible de te tenir devant les fourneaux, ou si tu y trouves un réel plaisir [...]. Un coup d'œil au réfrigérateur peut permettre de recueillir d'autres données, et précieuses : dans le compartiment à œufs, il ne reste qu'un œuf; en fait de citron, il n'y en a que la moitié d'un, et encore à moitié $\mathrm{sec}$ : on relève en somme une certaine négligence pour ce qui touche aux réapprovisionnements essentiels. Mais pour compenser, il y a de la crème de marron, des olives noires, un pot de salsifis; lorsque tu fais les courses, il est manifeste que tu te laisses attirer par les marchandises que tu vois exposées, au lieu de penser à ce qui manque à la maison. Ainsi, de l'observation de ta cuisine, peut-on, retirer l'image d'une femme extravertie et lucide, sensuelle et méthodique, qui met son sens pratique au service de sa fantaisie. » (Si par une nuit d'hiver un voyageur, Paris, Seuil, 1995, p. 161-162).

16. Philippe Hamon, Du Descriptif, Paris, Hachette, 1993, p. 33. 
transgression, transgression au regard des usages types de la cuisine. Que fait-on dans une cuisine dès lors que l'on n'y mange pas et que l'on n'y cuisine pas? C'est, semble-t-il, ce « coin aveugle » de la cuisine qui intéresse la littérature au premier chef. Si la cuisine est normalement, dans la maison, un lieu de vie essentiellement consacré à la préparation des mets et éventuellement à leur consommation, quand la description de la cuisine s'étoffe « anormalement » dans le roman, cette transgression, ce premier écart avec la norme annoncent généralement un usage atypique, voire transgressif de la cuisine. C'est tantôt un habitacle sans vie, qui enferme le personnage, l'étouffe, tantôt le lieu des amants, du criminel, voire du cannibale.

Faire l'amour dans la cuisine, Bob Rafelson avait su exploiter ce fantasme dans son adaptation du roman de James Cain, The Postman Always Rings Twice (Le Facteur sonne toujours deux fois), en 1981. À Lana Turner, parée de blanc pour incarner le personnage de Cora dans la version de Tay Garnett (1946), il substituait Jessica Lange, actrice dont la sensualité provocante éclatait sur l'écran dans une scène à l'érotisme torride. Malgré mes recherches, je n'ai pas trouvé d'équivalent dans le roman, la cuisine y est souvent un lieu où le corps des femmes est violenté, scènes rapides, brutales qui n'entraînent guère de description. Dans Pot-Bouille de Zola, Octave possède brutalement Marie sur la table de la cuisine « entre [une] assiette oubliée [où traînaient encore des queues de radis] et [un] roman, qu'une secousse fit tomber par terre ${ }^{17}$. La scène est encore plus crue dans The Hiawatha (Comme un frère), roman de David Treuer. Dans le spectacle de la femme renversée sur la table se dessine, par le biais du jeu métaphorique, l'image d'un animal blessé, atteint d'une balle au ventre, à l'œil vide :

"Here's fine, » he heard the man say. There was no reply from Betty, and then Simon heard spoons clatter and a thump that sounded like a body falling. [...] He had put her over the kitchen table. Betty endured. Simon could see one of her eyes, cool and unblinking, like a gut shot due, alive but too weak to stand. The table wobbled on its chrome legs, but it held. So she did. ${ }^{18}$

Dans ces scènes n'émerge aucune érotique de l'espace. La transgression ne se dit pas dans l'amplitude donnée à la séquence, mais dans la violence de l'acte et, surtout, dans sa visibilité let ce, en particulier chez Treuer, où le regard de l'observateur a dans son champ l'un des yeux de la femme, « froid

17. Émile Zola, Les Rougon-Macquart, Pot-Bouille, Paris, Gallimard, «Bibliothèque de la Pléiade », t. III, 1964, p. 76.

18. David Treuer, The Hiawatha, New York, Picador, 1999, p. 165 ; pour la traduction française de Marie-Claire Pasquier : " "Ici, ça ira", entend-il l'homme dire. Il n'y a pas de réponse, puis Simon entend un cliquetis de cuillers, et un bruit sourd comme un corps qui tombe. [...] Rojta l'avait renversée sur la table de la cuisine. Betty subissait. Simon voyait l'un de ses yeux, froid, immobile, comme une biche qu'on a atteinte au ventre, vivante mais trop faible pour se tenir debout. La table branlait sur ses pieds chromés, mais elle tenait le coup. Betty aussi. » (Comme un frère, Paris, Albin Michel, 2002, «Terres d'Amérique », p. 186). 
et immobile »). Ici est aboli le dualisme traditionnel des cultures «puritaines », où «l'Eros est repoussé dans les zones d'ombres qui sont aussi bien les secteurs cachés (quartiers ou pièces) que les dessous du visible. ${ }^{19}$

Contrairement à ces exemples, dans les romans où j'ai trouvé des descriptions de cuisines vraiment atypiques, j'ai constaté que, le plus souvent, ces descriptions n'intervenaient jamais seules, mais fonctionnaient plutôt en miroir, opposant de façon antithétique un type de cuisine à un autre, comme si cette inscription dans un duo signalait leur caractère surdéterminé. Dans la plupart de ces descriptions, ce lieu du familier, du « heimlich » par excellence qu'est la cuisine, soit un lieu qui renvoie généralement au foyer, au bonheur familial, se renverse en un lieu inquiétant, figure d'unheimlich, d'inquiétante étrangeté.

Dans Little, roman de David Treuer encore, on assiste ainsi à la description de deux cuisines parfaitement antithétiques. Tout d'abord, celle de Paul, nouveau prêtre nommé dans la petite ville de «Pauvreté », qui découvre sa demeure : le presbytère qui lui a été attribué. Alors que, comme le rappelle le texte «dans les constructions du Middle West, c'était la cuisine qui, à sa modeste échelle, était le centre d'attraction autour de quoi tout s'aimantait, vers quoi tout convergeait $»^{20}$, la cuisine agresse le nouveau venu par sa blancheur immaculée :

Finally, after having found the light switch nestled behind the slightly humming GE refrigerator, Paul clicked it on and saw the kitchen for the first time. The sight almost blinded him: everything was white.

The linoleum was sparkling white [...] The wooden cupboards had all been painted white too. It looked like one fresh unbroken coat but it was actually the twenty-first time they had been painted. [...] The aging wood was smooth and unbroken underneath a score of applications, the last of which was a dewy white.

The refrigerator was white inside and out [...]. There were no smells or stains, no scrapes, no marks. [...]

The four chairs that surrounded the Formica table were wooden, and they looked to Paul as if they had been painted at the same time as the cupboards. White, of course. ${ }^{21}$

19. Raymond Ledrut, «L'homme et l'espace », in : Histoire des mœurs, sous la direction de Jean Poirier, vol. 1, Paris, Gallimard, « Folio Histoire », 1990, p. 89.

20. David Treuer, Little [1995], Londres, Granta Book, 1997, p. 146; traduction française de Marie-Claire Pasquier : Little, Paris, Albin Michel, «10/18», 1998, p. 194.

21. Ibid., p. 146-147; trad. : «Finalement, après avoir découvert le commutateur niché derrière le réfrigérateur General Electric qui émettait un faible bourdonnement, et l'avoir actionné, Paul put voir la cuisine. Il en fut presque aveuglé : tout était blanc. Le linoléum était d'un blanc étincelant [...] Les placards aussi avaient tous été peints en blanc. On aurait dit une couche toute neuve, uniforme, mais c'était en fait la vingt et unième fois qu'ils étaient repeints. [...] Sous toutes ces couches successives, le bois vieillissant était intact et lisse, et la dernière couche était d'un blanc laiteux. Le réfrigérateur était blanc, dehors comme dedans. [...] Il n'y avait ni odeurs, ni taches, ni éraflures, ni marques. [...] Les quatre chaises qui entouraient la table en Formica étaient 
Ce décor transforme la cuisine en un lieu inhospitalier, austère, vide et nu. Bien que Paul, dans son rêve communautaire, se soit imaginé que « les gens viendraient se réunir dans sa cuisine pour discuter de leurs affaires personnelles », personne ne pénétrera jamais de son plein gré dans ce lieu sans vie, où toute trace d'activité humaine semble avoir mystérieusement été effacée. C'est un lieu d'étouffement oppressant, qui ne peut qu'accentuer le sentiment de solitude du personnage. En revanche, dans un autre passage, à cette description de cuisine dysphorisante fait écho la version euphorisante, quasi féerique d'une autre cuisine. Tandis que la jeune Violet a passé une partie de la matinée à balayer le sol de la cuisine, Lyle est venu sur ces entrefaites lui emprunter du sucre. Bien vite le trouble a submergé les deux jeunes gens:

As I turned around our legs tangled because we both went down on the floor together, my dress flew around us like a fine dust.

[...] My dress covered everything in the sunlight. It covered the wetness and trapped our heat. Faster. I was silk, and bolts of my red cloth unrolled and fluttered around us in a wind. Faster. He pulled away. As he jumped to his feet his pants came up with one hand while the other took the sugar. Grasping his pants and the sugar he ran out the door and into the sun.

I sat there on the floor awhile. Everything was still. Now, though, I picked up a faint pulse. From the sunlight, or the river maybe. [...] I looked at the floor, clean and hard in the morning sun. It looked different now. It looked too bare, naked. [...] I took the broom and spread the dirt back out across the floor. I flung it in some places, puffs of dust streaking the sunlight. I put some dirt back under the stove and pushed some under the table. I fanned it out in front of the doorway and when I was done, the floor was so much better that I finally felt that I could sit and let the sounds start up again. ${ }^{22}$

en bois, et Paul eut l'impression qu'elles avaient été peintes en même temps que les placards. En blanc, naturellement ». (Op. cit., p. 194-195).

22. Ibid., p. 176-177; trad. : «Pendant que je me retournais, nos jambes se sont emmêlées, parce qu'on est tous les deux tombés en même temps, et ma robe voletait autour de nous comme de la poussière fine. [...] Ma robe recouvrait tout, dans la lumière. Elle recouvrait la moiteur et emprisonnait notre chaleur. Plus vite. J'étais en soie, et la toile rouge de ma robe tourbillonnait autour de nous, prise dans la bourrasque. Plus vite. Il s'est dégagé. Il s'est remis sur pied d'un bond, remontant son pantalon d'une main, attrapant la boîte de sucre de l'autre. Il est parti en courant au soleil, sans lâcher ni le sucre, ni le pantalon. Je suis restée assise par terre un moment. Rien ne bougeait. Et puis j'ai perçu comme une palpitation dans l'air. Ça venait peut-être du soleil, ou de la rivière. [...] j'ai regardé le plancher, propre et dur dans la lumière du matin. Je ne le voyais plus de la même façon. Il me paraissait trop nu. [...] J'ai pris le balai, j'ai étalé la poussière sur tout le plancher. J'en ai lancé ici et là, ça faisait des gros flocons qui miroitaient au soleil. J'en ai remis sous le poêle, j'en ai glissé sous la table. J'en ai dispersé devant la porte et, quand j'ai eu fini, le plancher avait tellement meilleure allure que j'ai compris que je pouvais enfin m'asseoir et laisser les bruits revenir. » $10 p$. cit., p. 235-236). 
L'aspect poétique de ce passage tient essentiellement à l'image de la poussière, et à la transformation dont elle fait l'objet. Si, dans un premier temps, la poussière est impitoyablement traquée par la jeune femme, après la scène d'amour, elle devient signe érotique et solaire qui métamorphose la cuisine en y inscrivant la trace lumineuse du bonheur fugitif de l'étreinte.

C'est dans un tout autre registre - ignoble et monstrueux - qu'interviennent les descriptions de cuisine dans American Psycho de Bret Easton Ellis. Ici, les deux descriptions de la cuisine du golden-boy qui se font écho évoquent les oppositions de type «diététique » repérées par Lévi-Strauss au sein des systèmes mythologiques ${ }^{23}$. Loin d'obéir à un système aussi articulé que celui des mythes analysés par l'ethnologue, on constate cependant que, dans ce roman, les deux descriptions de la cuisine mettent en vis-à-vis les deux régimes alimentaires les plus éloignés au sein de l’arborescence mythologique élaborée par Lévi-Strauss: d'un côté, la cuisine luxueuse et aseptisée du végétarien et, de l'autre, la cuisine monstrueuse, lieu de l'immonde et du cannibale.

Au début du roman, le protagoniste est décrit de façon assez conventionnelle dans son décor matinal. Le style est caractéristique des romans d'Ellis, saturé de noms de marques et d'articles, censés traduire l'ennui culturel qui caractérise le consommateur de la fin du XXe siècle. Chez le «yuppie», la cuisine «a perdu sa fonction culinaire pour devenir un laboratoire fonctionnel ${ }^{24}$ à l'esthétique aussi raffinée que le régime auquel s'astreint le personnage :

I take a bran muffin, a decaffeinated herbal tea and a box of oat-bran cereal from one of the large glass-front cabinets that make up most of an entire wall in the kitchen; complete with stainless-steel shelves and sandblasted wire glass, it is framed in a metallic dark gray-blue. I eat half of the bran muffin [...] A bowl of oat-bran cereal with wheat germ and soy milk follows; another bottle of Evian water and a small cup of decaf tea after that. Next to the Panasonic bread baker and the Salton Pop-Up coffee maker is the Cremina sterling silver espresso maker [...] and the Sharp Model R-1810A Carousel II microwave oven with revolving turntable which I use when I heat up the other half of the bran muffin. Next to the Salton Sonata toaster and the Cuisinart Little Pro food processor and the Acme Supreme Juicerator and the Cordially Yours liqueur maker stands the heavy gauge stainless-steel two-and-onehalf-quart teakettle [...]. For what seems like a long time I stare at the Black \& Decker Handy Knife that lies on the counter next to the sink, plugged into the wall : it's a slicer/peeler with several attachments, a serrated blade, a scalloped blade and a rechargeable handle. ${ }^{25}$

23. Voir Claude Lévi-Strauss, Mythologiques, t. 2, Du Miel aux cendres, Paris, Plon, 1966, p. 29-30.

24. Jean Baudrillard, Le Système des objets, op. cit., p. 64.

25. Bret Easton Ellis, American Psycho [1991], Londres, Picador, 2006, p. 27-28; traduction française d'Alain Défossé : «Je prends un petit pain au son, un sachet de thé décaféiné et un paquet de céréales (avoine et son) dans un des vastes placards vitrés qui recouvrent presque tout le mur de la cuisine; le cadre est en métal gris-bleu soutenu, les étagères en inoxydable, et les panneaux en verre armé dépoli. Je mange la 
Dans cet intérieur, un signe tend à se détacher. Placé en fin de description, il provoque une pause chez le narrateur (« durant ce qui me paraît être un long moment, je contemple le couteau électrique »). Pour l'œil du lecteur averti, il s'agit d'un indice. C'est en effet cet ustensile qui servira ultérieurement de principal axe de renversement, pour produire une autre lecture de certains accessoires présentés comme anodins, voire inutiles dans cette première séquence. Décor d'une première nature morte sanglante ${ }^{26}$, la cuisine chic et sophistiquée de Patrick Bateman se transforme, un peu plus loin encore dans l'intrigue, en un lieu «gore », passage dont la lecture est à peine soutenable :

In the kitchen I try to make meat loaf out of the girl but it becomes too frustrating a task and instead I spend the afternoon smearing her meat all over the walls, chewing on strips of skin I ripped from her body [...]. The head in the microwave is now completely black and hairless and I place it in a tin pot on the stove in an attempt to boil any remaining flesh [...]. The smell of meat and blood clouds up the condo until I don't notice it anymore. [...] Maggots already writhe across the human sausage, the drool pouring from my lips dribbled over them, and still I can't tell if I'm cooking any of this correctly, because I'm crying too hard and I have never really cooked anything before. ${ }^{27}$

«Entre la personne sociale et son corps où la nature se déchaîne, entre ce corps même et l'univers biologique et physique, les ustensiles de tables [...] remplissent un rôle efficace au titre d'isolants ou de médiateurs. Leur présence interposée empêche la décharge catastrophique qui ris-

moitié du petit pain au son [...]. Suit un bol de céréales (avoine et son) avec germes de blé et lait de soja; encore une bouteille d'Evian, et une petite tasse de thé décaféiné pour finir. Alignés à côté du four à pain Panasonic et de la cafetière Salton, se trouvent le percolateur Cremina en argent massif [...] et le four à micro-ondes Sharp modèle R-1810A Carousel II à plateau tournant, dans lequel je fais réchauffer l'autre moitié du petit pain. À côté du grille-pain Salton Sonata, du robot Cuisin'art Little Pro, de la centrifugeuse Acme Supreme Juicerator, et du shaker électronique Cordially Yours, est posée la bouilloire à thé en inox [...]. Durant ce qui me paraît être un long moment, je contemple le couteau électrique Handy Knife de Black \& Decker, posé sur le plan de travail, à côté de l'évier, et branché sur la prise murale : c'est un éminceur/éplucheur doté de divers accessoires, avec une lame-scie et une lame dentée. » (American psycho, Paris, Salvy Éditeur, « Points », 1992, p. 42-43).

26. «Sa main gauche, tronçonnée, est posée crispée, sur le plan de travail de la cuisine, dans une petite flaque de sang, et sa tête sur la table. » (/bid., p. 374).

27. Ibid., p. 332 ; trad. : « Dans la cuisine, je tente de préparer un pâté avec la viande de la fille, mais cette tâche s'avère vite ingrate, et je passe l'après-midi à l'étaler partout sur les murs, tout en mâchant des lambeaux de peau arrachés au corps [...]. Dans le four à micro-ondes, la tête est maintenant complètement noire et chauve, et je la mets à bouillir dans une casserole sur le fourneau [...]. L'odeur de la viande et du sang envahit l'appartement, à tel point que je ne la remarque même plus. [...] Déjà, les asticots se tortillent sur la saucisse humaine, et la bave qui s'écoule de ma bouche goutte sur eux; je ne sais si je prépare cela correctement, parce que je pleure trop fort, et que je n'ai jamais vraiment fait la cuisine auparavant. » (Op. cit., p. 443-444). 
querait de se produire ${ }^{28}$, écrit Lévi-Strauss. C'est bien cette « décharge catastrophique » à laquelle nous assistons ici. La liturgie du repas s'inverse en une cuisine morbide et solitaire, qui détruit les frontières entre les catégories traditionnelles que sont nature et culture, cru et cuit, bouilli et pourri. Alors que normalement, « la vaisselle permet d'éloigner le monde ingérable du sale et d'éviter au mangeur d'être sali par lui ${ }^{29}$, ici la souillure se diffuse de façon envahissante. L'espace ne rend plus la nourriture désirable, mais reflète dans sa détérioration le déséquilibre mental du serial-killer. Le mangeur est seul, hors rituel, plus aucune prescription ou prohibition ne règle sa conduite; plus aucune médiation technique ne modère cet échange entre soi et le monde qu'est la nutrition. L'extrême sophistication de Patrick Bateman s'inverse en pure sauvagerie, libérée de toute forme de sociabilité domestique.

$\mathrm{Si}$, dans la plupart des cas de transgressions rencontrés, les descriptions de cuisines fonctionnent en série ou en duo, la cuisine familiale de Mort à crédit fait exception à la règle. Chez Céline, la cuisine est un lieu qui d'emblée suggère la transgression. Dans Voyage au bout de la nuit déjà, on se souvient du passage où Bardamu reconstitue, depuis son appartement, la scène horrible qui résonne dans l'arrière-cour : un couple attache et frappe son enfant pour s'exciter. «C'était ainsi qu'ils faisaient l'amour tous les deux que m'a expliqué leur concierge, dans la cuisine ça se passait contre l'évier. Autrement, ils y arrivaient pas $\gg{ }^{30}$. Oserai-je la comparaison ? Autant chez Philippe Delerm, le «chronotope ${ }^{31}$ de la cuisine tient lieu de forme localisée du bonheur, de l'intimité heureuse, autant chez Céline la mièvrerie du bric-à-brac bienheureux dépeint par Delerm explose pour traduire le capharnaüm infernal où se déchaîne la violence familiale. Rien ne s'immisce chez Céline de la douceur de vivre qui, chez Delerm, fait écho aux toiles de Carl Larsson :

Quand nous sommes arrivés dans la maison, les murs de la cuisine étaient de ce vert pâle d'hôpital qui semble appeler le néon, le tremblement blafard sur un espace vide et cru. La couleur maintenant, c'est yaourt au cassis, un mauve chaud qui réduit les distance et nous rapproche du terrier. La rampe de néon est montée au grenier. Une suspension d'osier l'a remplacée, dessine un cercle où la vie se confine, invente des frontières et blondit le bois pâle.

28. Claude Lévi-Strauss, L'Origines des matières de table, Mythologies, t. III, Paris, Plon, 1968, p. 420.

29. Frédéric Lange, Manger ou les jeux et les creux du plat, Paris, Seuil, 1975, p. 38.

30. Louis-Ferdinand Céline, Voyage au bout de la nuit, Paris, Gallimard, 1952, « Folio », p. 339.

31. L'expression est de Mikhaïl Bakhtine (voir Esthétique et théorie du roman, Paris, Gallimard, «Tel», 1975/1978, p. 237). Je l'emploie cependant ici dans une acception plus large, telle que la définit Jacques Dubois dans Les Romanciers du réel : «il s'agit, dans un corpus romanesque donné, de sites de prédilection où s'exprime un rapport singulier au temps et à l'existence » (op. cit., p. 86). 
Mais c'est au vaisselier qu'on reconnaît surtout notre penchant pour la vie des souris anglaises. Sur trois étages resserrés, c'est un fourmillement de choses à manger pour les yeux. Ce sont les boîtes qui dominent : l'hilarité statique du bonhomme Banania à chéchia rouge sur fond de désert jaune-orange voisine avec le flegme en médaillon du très britannique Sir Thomas Adam's dont le profil sobre à moustache cautionne le sérieux des celebrated old english Oatcakes... ${ }^{32}$

Dans Mort à crédit, la cuisine est dépourvue de ses caractéristiques traditionnelles, ce qui signale d'emblée la tension familiale. Comme l'écrit en effet Baudrillard: «chaque pièce a une destination stricte qui correspond aux diverses fonctions de la cellule familiale, et plus loin renvoie à une conception de la personne comme d'un assemblage équilibré de facultés distinctes $»^{33}$. Chez Céline, l'équilibre est toujours précaire, véritable chaos, « déluge en suspens »:

On éteignait la boutique. Ma mère était pas cuisinière, elle faisait tout de même une ratatouille. Quand c'était pas «panade aux œufs » c'était sûrement «macaroni ». Aucune pitié. Après les nouilles on restait un moment tranquilles, à réfléchir pour l'estomac. [...] Là derrière la tapisserie c'était l'éclairage papillon. Il faisait obscur dans les assiettes. Ma mère elle reprenait des nouilles, stoïque, pour nous inciter... Il fallait une bonne gorgée de vin rouge pour s'empêcher de les vomir.

Le réduit des repas, il servait en plus pour la lessive et pour garer les rogatons... Y en avait des monceaux, des piles... Ceux qu'étaient pas rafistolables, les invendables, les pas montrables, les pires horreurs. Du vasistas pendaient des toiles dans la soupe. [...]

Un décor de musée sale. [...]

Pour pas perdre de vue sa montre [mon père] l'accrochait dans la cuisine au-dessus des nouilles. [...] Elle alors mettait la table. Elle foutait une assiette en l'air. Il s'emportait, se ruait au secours. C'était si petit dans notre piaule qu'on butait partout. $Y$ avait jamais de place pour un furieux dans son genre. La table elle carambolait, les chaises entraient dans la valse. C'était une pagaye affreuse. Ils trébuchaient l'un dans l'autre. Ils se relevaient pleins de ramponneaux. ${ }^{34}$

Si la description de la cuisine ne donne pas lieu chez Céline à deux versions antithétiques, c'est, semble-t-il, parce que « dans la scène routinière, les indices d'une autre scène sont dispersés » ${ }^{35}$. Avant même que la bagarre n'éclate dans sa violence meurtrière entre les parents, elle est déjà inscrite dans ce décor de chaos, qui reflète un ordre familial instable dont la saturation peut à tout moment déclencher l'explosion.

32. Philippe Delerm, Le Bonheur, Tableaux et bavardages, Paris, Éditions du Rocher, 1986/1998, p. 121-122

33. Jean Baudrillard, Le Système des objets, op. cit., p. 21.

34. Louis-Ferdinand Céline, Mort à crédit, Paris, Gallimard, «Folio », 1952, p. 58-67.

35. Jacques Dubois, Les Romanciers du réel, op. cit., p. 97. 
À l'exception de la scène de bonheur fugitive entre les deux amants, les autres cuisines qui sont décrites traduisent des figures de dérèglement, qui remettent en cause l'image de la cuisine comme «lieu intime ${ }^{36}$, propre à la maison moderne. Dans les trois cas, ce sont des «anti-cuisines » qui sont dépeintes: blancheur ascétique de la cuisine du prêtre qui semble le condamner à une abstinence tant charnelle qu'alimentaire, lui interdire toute forme d'intimité bienheureuse avec son propre corps, et cuisines de l'« ensauvagement » chez Céline et Ellis. Dans ces trois exemples, l'équilibre biologique et corporel, qui assure l'intégrité de l'être humain, est menacé. Au sein de la cuisine, espace qui se veut éminemment réglé et culturalisé, les « configurations plus ou moins déterminées » qu'entretiennent entre eux les objets ne sont plus respectées. Des toiles dans la soupe, une montre au-dessus des nouilles, une tête chauve et noircie dans le four à micro-ondes ou, à l'inverse, une absence de rapport entre objets, formes et couleurs dans la cuisine de Paul, ces différents éléments montrent que l'ordre de la cuisine s'est effondré alors que, normalement, « chaque objet a une place ou des places différentes en relation avec celles des autres objets, et [que] les changements de place obéissent à des règles plus ou moins précises de déplacement et de remplacement $\gg{ }^{37}$. Pourquoi une telle rigidité de l'ordonnancement? La cuisine, on le sait, entretient des relations de proximité extrême avec la mort et la sexualité ${ }^{38}$. Elle doit donc se garder de tous les débordements libidinaux qu'une telle contiguïté peut susciter. Son ordre tient lieu de « cordon sanitaire » destiné à assurer la survie du corps - et, si possible, son plaisir. À cet effet, les échanges y sont strictement réglés, respectant les compatibilités culturellement admises. Dans la cuisine s'élaborent poulets, rôtis et grillades, dont la préparation rend presque oublieux de leur nature de chairs mortes. Son origine plus ou moins refoulée, la viande rendue propre à la consommation est supposée fortifier les corps, célébrer leur réunion et leur réconciliation autour d'un plat. En revanche, l'espace qui, dans sa vacuité blanche, rend obscène toute nourriture, l'espace qui suscite des pulsions de meurtre, ou encore l'espace qui consacre la détestation de son propre corps ne peuvent être figurés que comme des anti-cuisines.

Crystel PINÇONNAT Université Paris-Diderot

36. Igor de Garine, «Introduction », Cuisines, reflets de sociétés, textes réunis et présentés par Marie-Claire Bataille-Benguigui et Françoise Cousin, Paris, Musée de l'Homme, Edition Sépia, 1996, p. 12.

37. Raymond Ledrut, « L'homme et l'espace », art. cité, p. 66.

38. Voir Jean Pouillon, "Manières de table, manières de lit, manières de langage », Nouvelle Revue de Psychanalyse, n6, automne 1972, p. 9-25; Luce Giard, Faire la cuisine, in : L'Invention du quotidien, t. 2, Habiter, cuisiner, Gallimard, «Folio/Essais », 1994 , p. 277-279. 